\title{
Penile metastasis secondary to nasal-type extranodal natural killer/T-cell lymphoma: A case report and review of the literature
}

\author{
YANAN LI ${ }^{1 *}$, XIAORUI FU ${ }^{1 *}$, JINGJING WU ${ }^{1}$, CHANG YU ${ }^{1}$, ZHAOMING LI $^{1}$, ZHENCHANG SUN $^{1}$, JIAQIN YAN ${ }^{1}$, \\ FEIFEI NAN ${ }^{1}$, XUNDONG ZHANG $^{1}$, LING LI ${ }^{1}$, XIN LI $^{1}$, LEI ZHANG ${ }^{1}$, WENCAI LI ${ }^{2}$, \\ GUANNAN WANG $^{2}$ and MINGZHI ZHANG ${ }^{1}$
}

${ }^{1}$ Lymphoma Diagnosis and Treatment Center, Department of Oncology; ${ }^{2}$ Department of Pathology, The First Affiliated Hospital of Zhengzhou University, Zhengzhou, Henan 450052, P.R. China

Received November 4, 2015; Accepted April 13, 2017

DOI: $10.3892 / \mathrm{ol} .2018 .8288$

\begin{abstract}
Extranodal natural killer/T-cell lymphoma (NKTL), nasal-type is one of the most aggressive lymphoid malignancies and is characterized by an extremely poor survival outcome. The present study reports the case of a 39-year-old Chinese male with history of extranodal NKTL who presented with a painless indurated mass in the glans penis. The results of an incisional biopsy revealed atypical cells that were positive for CD3, CD56, T-cell-restricted intracellular antigen-1, granzyme B and Epstein-Barr virus-encoded RNA, and negative for CD20. A diagnosis of metastatic NKTL was determined. The patient was treated with systemic chemotherapy consisting of cisplatin, dexamethasone, gemcitabine and pegaspargase, which resulted in remission and regression of the mass. In addition, a review of the literature was performed, and the data for 13 cases of non-B-cell penile lymphoma, including the present case, are presented. To the best of our knowledge, this is first review of this entity.
\end{abstract}

\section{Introduction}

Extranodal NK/T-cell lymphoma (NKTL) is a highly aggressive neoplasm that commonly involves the nasal cavity, which accounts for $1-10 \%$ of all non-Hodgkin's lymphoma (1). The estimated 5-year overall survival rate is $40-50 \%$ (2). This type of tumor has distinct clinicopathological features and no standard treatment options. In the early stages, the disease often presents as a destructive lesion within the centrofacial region. However, in advanced cases, the disease may involve distant extranodal organs, frequently including the skin, liver, lung, testis or central nervous system $(1,3)$. Penile involvement,

Correspondence to: Professor Mingzhi Zhang, Lymphoma Diagnosis and Treatment Center, Department of Oncology, The First Affiliated Hospital of Zhengzhou University, 1 Jianshe Road, Zhengzhou, Henan 450052, P.R. China

E-mail: mingzhi_zhang1@163.com

*Contributed equally

Key words: natural killer/T cell lymphoma, penis, immunohistochemistry, chemotherapy, pegaspargase, metastasis whether primary or secondary, is extremely rare and seldom reported in the literature $(4,5)$. Early diagnosis of lymphoma can aid in avoidance unnecessary penile amputation and allow for optimal management (6). The first English-language case report of extranodal NKTL with involvement of the penis was reported in 2008, and is the only study in English to describe this entity (7).

The present study reports the comprehensive clinical data of one case of extranodal NKTL with recurrence in the penis, and reviews the previously reported cases of non-B-cell penile lymphoma. Written informed consent for the publication of this report was obtained from the patient.

\section{Case report}

Ethical approval was obtained from the Scientific Research and Clinical Trial Ethics Committee of The First Affiliated Hospital of Zhengzhou University (Zhengzhou, China) and written informed consent was obtained from the patient. A 39-year-old Chinese male presented to The First Affiliated Hospital of Zhengzhou University (Zhengzhou, China) in February 2013 complaining of nasal congestion and purulent rhinorrhea that had lasted for 6 months. Upon physical examination, a mass was observed in the right nasal cavity, and an enlarged palpable lymph node was detected in the ipsilateral neck. A subsequent nasopharyngeal computed tomography (CT) scan revealed a right nasal tumor. Subsequently, an incisional biopsy of the right mass was performed. Tissue was fixed in $10 \%$ buffered formalin for $6 \mathrm{~h}$ at $37^{\circ} \mathrm{C}$, dehydrated, embedded in paraffin and cut into $4-\mu \mathrm{m}$-thick sections. The sections were blocked in $3 \%$ hydrogen peroxide for $4 \mathrm{~min}$ at $37^{\circ} \mathrm{C}$ before incubation with antibodies. The primary antibodies used were CD56 (cat no. 56C04; Thermo Fisher Scientific, Inc., Waltham, MA, USA) in 1:200 dilution, CD3 (cat no. Kit-0003; MXB Biotechnologies, Inc., Fuzhou, China) in undiluted solution, granzyme B (cat no. ZA-0149; OriGene Technologies, Inc., Beijing, China) in undiluted solution, T-cell-restricted intracellular antigen-1 (TIA-1; cat no. MAB-0576; MXB Biotechnologies, Inc.) in undiluted solution, CD43 (cat no. MAB-0032; MXB Biotechnologies, Inc.) in undiluted solution, CD20 (cat no. ZM-0039; OriGene Technologies, Inc.) in 1:200 dilution and Ki-67 (cat no. Kit-0005; MXB Biotechnologies, Inc.) in undiluted solution. The section specimens were incubated with 
primary antibodies against CD56, CD3, TIA-1, CD43, CD20 or Ki67 for 36, 72, 64, 36, 36 or $64 \mathrm{~min}$ at $37^{\circ} \mathrm{C}$ respectively, then incubated with ultraView Universal DAB Detection kit (cat no. 760-500; Roche Diagnostics, Shanghai, China) that included anti-mouse and -rabbit secondary antibodies conjugated with horseradish peroxidase and DAB in undiluted solutions at $37^{\circ} \mathrm{C}$ for $16 \mathrm{~min}$. Images were taken with a Leica DM4 B microscope (x400 magnification; Leica Microsystems $\mathrm{GmbH}$, Wetzlar, Germany). The biopsy revealed atypical lymphocytes that were positive for CD56, CD3, granzyme B, TIA-1and CD43, and negative for CD20. Positive Ki-67 antigen staining, representing the proliferative activity, was identified in $70 \%$ of the cells. A routine ultrasound scan showed an enlarged lymph node, measuring $16 \times 6 \mathrm{~mm}$, in the right neck. Abdominal and pelvic ultrasound examinations were negative, and bone marrow smear and blood tests were unremarkable. These findings led to a diagnosis of NKTL stage IIE (involvement of nasal cavity and ipsilateral lymph nodes) according to the Ann Arbor classification (8), and the International Prognostic Index (IPI) (9) score was 1.

The patient received radiotherapy by radioisotope ${ }^{60} \mathrm{Co}$ (200 cGy, 25 times; total, 5,000 cGy) followed by seven cycles of chemotherapy, consisting of cyclophosphamide $\left(750 \mathrm{mg} / \mathrm{m}^{2}\right.$; day 1$)$, doxorubicin $\left(25 \mathrm{mg} / \mathrm{m}^{2}\right.$; days 1 and 2$)$ and vincristine (2 mg; day 1), with fotemustine $\left(100 \mathrm{mg} / \mathrm{m}^{2}\right.$; day 3 ) for the first 5 cycles. Each cycle lasted 21 days. A complete remission was achieved, and the patient was disease-free for 16 months.

Subsequently, the patient presented with a painless indurated mass in the glans penis that had been present for 1 week. No fever, weight loss or night sweats were reported. The patient denied having had unprotected sexual intercourse, previous sexually transmitted diseases or recent trauma. The physical examination revealed a firm mass affecting the glans penis, without ulceration or swelling, and multiple enlarged palpable lymph nodes in the bilateral inguinal regions. The results of a blood test and biochemical analyses, including an analysis of Epstein-Barr virus (EBV) DNA copies, were in the normal ranges. Enhanced CT showed a soft tissue mass with obscure boundaries and moderate enhancement on the arterial phase, and enlarged inguinal lymph nodes with ring-like enhancement (Fig. 1). An incisional biopsy of the penis was performed as described previously, which led to a diagnosis of penile metastasis secondary to NKTL. Immunohistochemical staining revealed that the biopsy tissue was positive for $\mathrm{CD} 3, \mathrm{CD} 56, \mathrm{CD} 43$, TIA-1, granzyme $\mathrm{B}$ and CD-30 (cat no. ZM-0043; OriGene Technologies, Inc.; Fig. 2). Additionally, in situ hybridization analysis revealed positive expression of EBV-encoded RNA (EBER; cat no. ISH-5021; OriGene Technologies, Inc.), the CD20 (Fig. 2) andanaplastic lymphoma kinase staining results were negative, and staining for Ki-67 was positive in 90\% of cells. Enhanced CT scans of the nasopharynx, chest and abdomen, an ultrasound scan of the superficial lymph node-bearing region, and bone marrow aspiration were performed, and no other lesions were detected. A diagnosis of recurrent extranodal NKTL was determined. The patient's clinical stage was IIE, and the IPI score was 1.

The patient then received three cycles of DDGP chemotherapy, consisting of cisplatin $\left(20 \mathrm{mg} / \mathrm{m}^{2}\right.$; days $\left.1-4\right)$, dexamethasone $\left(15 \mathrm{mg} / \mathrm{m}^{2}\right.$; days $\left.1-5\right)$, gemcitabine $\left(0.8 \mathrm{~g} / \mathrm{m}^{2}\right.$; days 1 and 8$)$ and pegaspargase $\left(2,500 \mathrm{IU} / \mathrm{m}^{2}\right.$; day 1$)$. The penile mass decreased in size following the first cycle and a partial remission was achieved. After two cycles of chemotherapy, the mass in the glans penis and the inguinal lymph nodes had regressed (Fig. 1). Following a further cycle of chemotherapy, the patient remained in a stable condition. Follow-up with the patient was not possible as contact was lost following the last chemotherapy treatment.

\section{Discussion}

Extranodal NKTL, nasal type is a type of lymphoma that associated with EBV, and is more commonly observed in Asian populations than in people of European descent $(1,3)$. This type of tumor has distinct clinicopathological features and no standard treatment options. In the early stages, the disease often presents as a destructive lesion within the centrofacial region. However, in advanced cases, the disease may involve distant extranodal organs, frequently the skin, liver, lung, testis, or CNS $(1,3)$. The present study reports a rare case of primary nasal extranodal NKTL with metastasis to the penis. In this patient, the tissue biopsy obtained from the penile mass exhibited atypical lymph cells infiltrating and damaging blood vessels, with considerable necrosis, and cells positive for CD56, EBER, granzyme B and TIA-1, and negative for CD20. The morphological characteristics and immunohistochemical findings of these cells were similar to those of primary nasal extranodal NKTL, and were thus consistent with a diagnosis of penile metastasis from nasal extranodal NKTL.

Penile malignancy is extremely rare, despite the rich blood supply to this region, with a reported incidence ranging from 0.58 to 1.3 cases $/ 100,000$ individuals $(4,5)$. Squamous cell carcinoma is the most common histological type $(91.7 \%)(4,5)$. Involvement of the penis by lymphoma is an even more rare occurrence. Diffuse large B-cell lymphoma is the most common histological subtype among cases of penile lymphoma (10). Sun et al (10) reported 33 cases of penile lymphoma, only 9 of which had originated from T-cells. In the current study, a search of PubMed with the terms 'lymphoma' combined with 'penis' or 'penile' identified only 12 cases of non-B-cell penile lymphoma that have been reported since 1995, of which two cases were ultimately diagnosed as NKTL. Four of these reports were in published in Chinese. The clinical characteristics of these cases are summarized in Table I (6,7,11-20).

The ages of the 13 patients (including the patient in the present case report) ranged from 29 to 77 years (median, 59 years). Of the 13 cases, 3 were extranodal NKTL, with 2 of these cases being metastatic tumors. In these 2 cases of metastatic NKTL, the time intervals between primary malignancy and recurrent penile lymphoma were 16 months and 5 years (7), and the primary lymphomas were located in nasal cavity in both cases. Following systemic chemotherapy, remissions were achieved in these 2 cases. While the majority of non-B-cell penile lymphoma cases respond to chemotherapy, 2 of the 13 patients reportedly succumbed to the disease.

The clinical presentations of penile lymphomas are often non-specific and subtle, and include nodules (11), masses $(7,12,13,16)$, ulceration $(6,11,13-15,17-20)$ and diffuse penile swelling $(13,14,19)$; more rare symptoms include dysuria and phimosis (18). Among the reviewed cases, 69\% ( $9 / 13$ cases) exhibited ulceration during the course of the disease, which may be a sign that indicates a possible diagnosis 

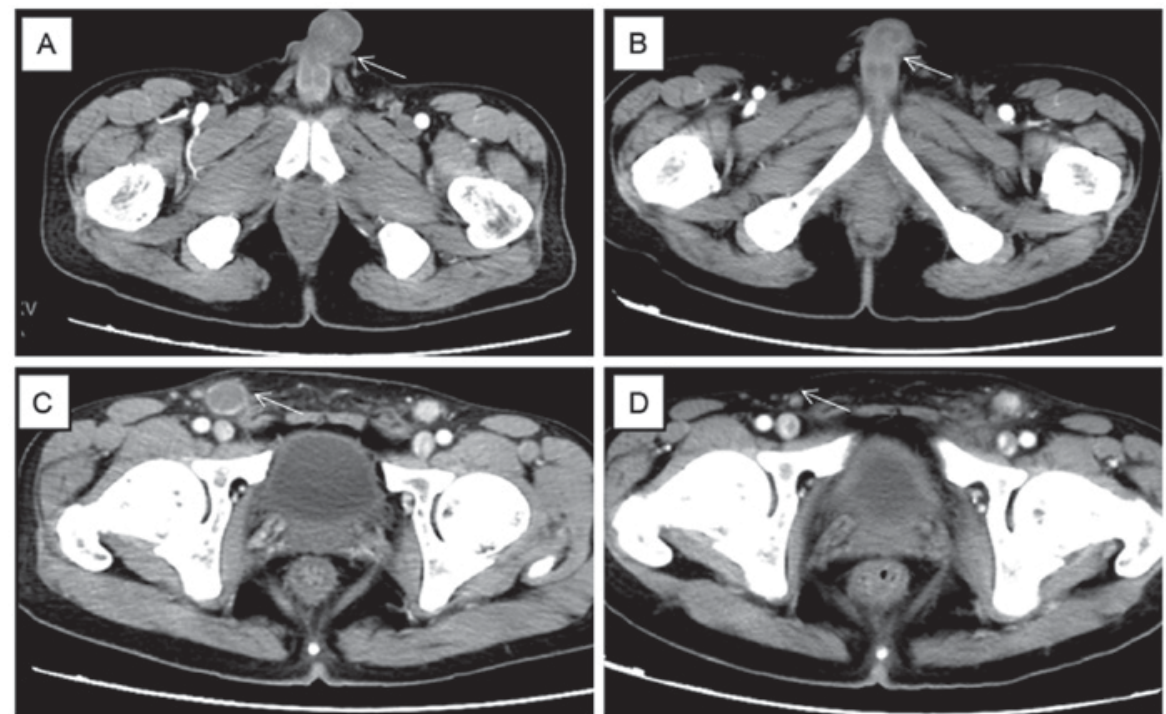

Figure 1. Computed tomography scans. (A) The arrow showed a soft tissue mass with obscure boundaries and moderate enhancement in the glans penis on the arterial phase. (C) The arrow showed an enlarged inguinal lymph node with ring-like enhancement. (B and D) The arrows showed the mass and the lymph node regressed following chemotherapy.
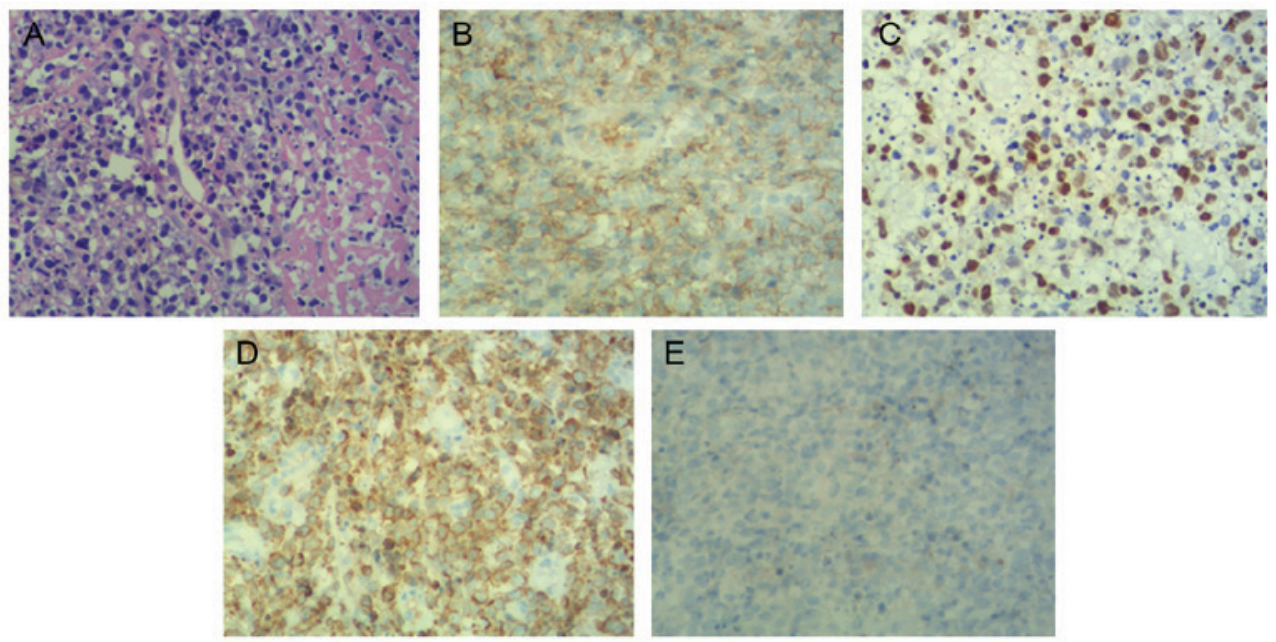

Figure 2. Microscopic findings. (A) Angiocentric and angioinvasive growth pattern of neoplastic cells with considerable necrosis (hematoxylin and eosin staining; magnification, $\mathrm{x} 400$ ). Tumor cells were positive for (B) CD56, (C) Epstein-Barr virus-encoded RNA (assessed by in situ hybridization), and (D) CD3, but negative for (E) CD20 (EnVision method; magnification, $\mathrm{x} 400$ ).

of penile lymphoma. Lymph node involvement was reported in $31 \%$ of cases (4/13 patients).

The diagnosis of metastatic penile NKTL can be challenging, and the time between presentation and a final diagnosis can be as long as 18 months (11). A major differential diagnostic challenge is the distinction between this entity and non-malignant necrosis; necrosis is common in diagnostic biopsies, and may significantly delay diagnosis. Biopsies must include the edges of lesions in order to increase the chance of having viable tissue. The differential diagnosis also includes vasculitis, trauma, and sexually transmitted diseases $(11,21)$. Therefore, a full personal history, physical examination and an incisional biopsy are essential to determine the correct diagnosis. Systemic radiological investigation, including CT, magnetic resonance imaging or positron emission tomography scans, as well as bone marrow aspiration or biopsy, should be undertaken.
Since metastases of nasal extranodal NKTL to the penis are rare, optimal treatment is not well established. Previous cases of lymphoma of the penis have been treated with chemotherapy $(7,15-17,19)$, chemotherapy combined with radiotherapy $(20)$, surgery $(14,18)$ or a combination of these treatment modalities $(6,11-13)$. Chemotherapy with or without radiotherapy is the optimal initial treatment to eradicate this type of tumor while preserving the physiology and function of the penis. Surgery should be reserved only for failures (17-20). In the present case, we opted to treat the patient aggressively with chemotherapy for the disseminated lesions. Furthermore, considering the patient's age, surgery or radiotherapy were not recommended due to possible disfigurement and loss of function. Selection of the optimal chemotherapeutic regimen is challenging due to the lack of published research, with no standard chemotherapy treatment established for NKTL, much less penile metastasis. Recent clinical studies have suggested that 


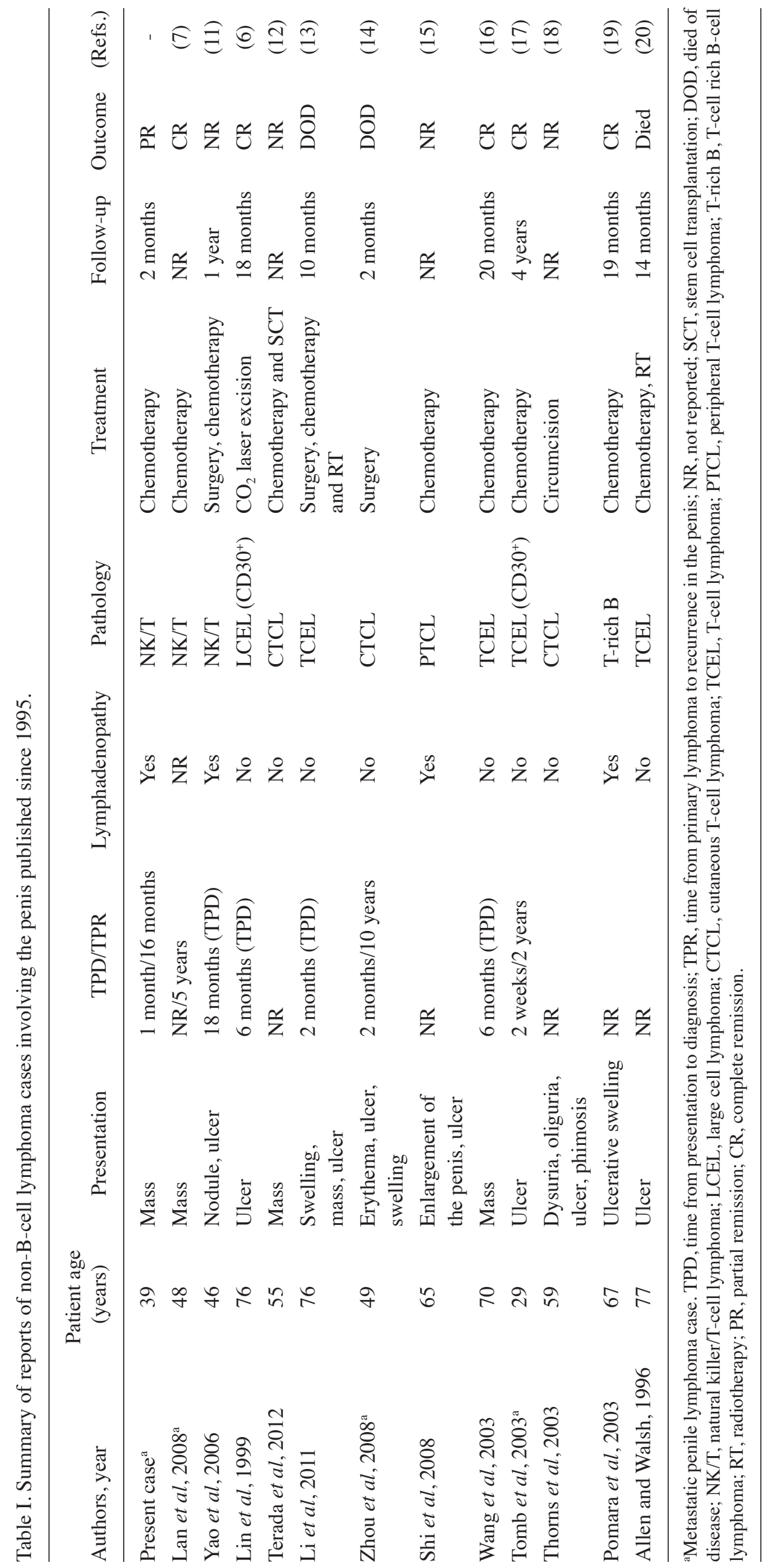


pegaspargase-based chemotherapy is a potential therapeutic option with a good remission rate for advanced NKTL $(22,23)$. In the current case, the patient received three cycles chemotherapy of DDGP (including cisplatin, dexamethasone, gemcitabine, pegaspargase), and the penile mass was decreased after the first cycle. After two cycles of chemotherapy, a partial remission was achieved. The patient subsequently received another cycle of chemotherapy and remained in stable condition.

In conclusion, penile metastasis secondary to extranodal NKTL without recurrence at the primary site, although rare, should be considered in the differential diagnosis of a penile mass. Pegaspargase-based chemotherapy may produce a positive outcome in such cases, at least in the short-term.

\section{Acknowledgements}

Not applicable.

\section{Funding}

The present study was supported by the National Natural Science Foundation of China (grant no. 81570203).

\section{Availability of data and materials}

All data generated or analyzed during this study are included in this published article.

\section{Authors' contributions}

YL and XF wrote the manuscript. JW, CY, ZL, ZS and JY performed the literature review. FN, XZ, LL, XL and LZ organized the figures and tables. WL and GW performed the pathologic diagnosis. MZ directed the diagnosis and management procedure of the patient and revised the manuscript.

\section{Ethics approval and consent to participate}

Ethical approval was obtained from the Scientific Research and Clinical Trial Ethics Committee of The First Affiliated Hospital of Zhengzhou University (Zhengzhou, China) and written informed consent was obtained from the patient.

\section{Consent for publication}

Written informed consent was obtained from the patient for publication.

\section{Competing interests}

The authors declare that they have no competing interests.

\section{References}

1. Haverkos BM, Pan Z, Gru AA, Freud AG, Rabinovitch R, Xu-Welliver M, Otto B, Barrionuevo C, Baiocchi RA, Rochford R and Porcu P: Extranodal NK/T cell lymphoma, nasal type (ENKTL-NT): An update on epidemiology, clinical presentation and natural history in North American and European cases. Curr Hematol Malig Rep 11: 514-527, 2016.

2. Suzuki R: Pathogenesis and treatment of extranodal natural killer/T-cell lymphoma. Semin Hematol 51: 42-51, 2014.
3. Au WY, Weisenburger DD, Intragumtornchai T, Nakamura S, Kim WS, Sng I, Vose J, Armitage JO and Liang R; International Peripheral T-Cell Lymphoma Project: Clinical differences between nasal and extranasal natural killer/T-cell lymphoma: A study of 136 cases from the international peripheral t-cell lymphoma project. Blood 113: 3931-3937, 2009.

4. Baldur-Felskov B, Hannibal CG, Munk C and Kjaer SK: Increased incidence of penile cancer and high-grade penile intraepithelial neoplasia in denmark 1978-2008: A nationwide population-based study. Cancer Causes Control 23: 273-280, 2012.

5. Barnholtz-Sloan JS, Maldonado JL, Pow-sang J, Giuliano AR and Guiliano AR: Incidence trends in primary malignant penile cancer. Urol Oncol 25: 361-367, 2007.

6. Lin DW, Thorning DR and Krieger JN: Primary penile lymphoma: Diagnostic difficulties and management options. Urology 54: 366, 1999.

7. Lan SK, Lin CW, Ho HC, Lee MS, Tzeng JE and Su YC: Penile metastasis secondary to nasal NK/T-cell lymphoma. Urology 72: 1014-1015, 2008.

8. Lister TA, Crowther D, Sutcliffe SB, Glatstein E, Canellos GP, Young RC, Rosenberg SA, Coltman CA and Tubiana M: Report of a committee convened to discuss the evaluation and staging of patients with Hodgkin's disease: Cotswolds meeting. J Clin Oncol 7: 1630-1636, 1989.

9. Lee J, Suh C, Park YH, Ko YH, Bang SM, Lee JH, Lee DH, Huh J, Oh SY, Kwon HC, et al: Extranodal natural killer T-cell lymphoma, nasal-type: A prognostic model from a retrospective multicenter study. J Clin Oncol 24: 612-618, 2006.

10. Sun J, Medeiros LJ, Lin P, Lu G, Bueso-Ramos CE and You MJ: Plasmablastic lymphoma involving the penis: A previously unreported location of a case with aberrant CD3 expression. Pathology 43: 54-57, 2011.

11. Yao HJ, Ying J, Wang Z, Yao DH, Ren XM and Bao YY: One case report of primary penile malignant lymphoma (with a review of 24 case reports). Zhonghua Nan Ke Xue 12: 520-524, 2006.

12. Terada T, Shirakashi Y and Sugiura M: T-cell lymphoma of the penis as the first manifestation of adult T-cell lymphoma/leukemia. Int J Dermatol 51: 973-975, 2012.

13. Li YL, Wang QZ, Ding GF, Li LX, Ni Z and Wang XM: Primary non-Hodgkin's lymphoma of the testis and penis: Clinical analysis of 5 cases. Zhonghua Nan Ke Xue 17: 254-256, 2011 (In Chinese).

14. Zhou ZL, Wang CY, Xu ZS and Zheng BZ: Primary cutaneous T-cell lymphoma of the penis complicated by Fournier gangrene: A case report. Zhonghua Nan Ke Xue 14: 542-544, 2008.

15. Shi YL, Yin HL, Zhou XJ, Zhou HB and Lu ZF: Primary peripheral T-cell lymphoma of the penis: A case report and review of the literature. Zhonghua Nan Ke Xue 14: 1003-1006, 2008 (In Chinese)

16. Wang HT, Lo YS and Huang JK: Primary lymphoma of the penis. J Chin Med Assoc 66: 379-381, 2003.

17. Tomb RR, Stephan F, Klein-Tomb L, Chahine G and Grosshans E: Recurrent primary CD30+ lymphoma of the penis. Br J Dermatol 149: 903-905, 2003.

18. Thorns C, Urban H, Remmler K, Dietel A, Lange K and Merz H: Primary cutaneous T-cell lymphoma of the penis. Histopathology 42: 513-514, 2003.

19. Pomara G, Cuttano MG, Tripodo C, Carlino F and Selli C: Primary T-cell rich B-cell lymphoma of the penis: A first case. BJU Int 91: 889, 2003.

20. Allen DC and Walsh MY: Malignant lymphoma of the scrotum and Wegener's granulomatosis of the penis-genital presentation of systemic disease. Ulster Med J 65: 169-172, 1996.

21. Yanagi H, Nakamura Y, Takagi D and Kubota K: Extranodal natural killer/T-cell lymphoma: A diagnostic dilemma. Rhinology 50: 325-331, 2012.

22. Zhou Z, Li X, Chen C, Li X, Zhang L, Li L, Wang X, Ma W, $\mathrm{Fu} \mathrm{X}, \mathrm{Wu} \mathrm{J}$, et al: Effectiveness of gemcitabine, pegaspargase, cisplatin and dexamethasone (DDGP) combination chemotherapy in the treatment of relapsed/refractory extranodal NK/T cell lymphoma: A retrospective study of 17 patients. Ann Hematol 93: 1889-1894, 2014.

23. Wen JY, Li M, Li X, Chen J, Lin Q, Ma XK, Dong M, Wei L, Chen ZH and Wu XY: Efficacy and tolerance of pegaspargase-based chemotherapy in patients with nasal-type extranodal NK/T-cell lymphoma: A pilot study. Asian Pac J Cancer Prev 15: 6275-6281, 2014. 